\title{
Escorias de alto horno: composición y comportamiento hidráulico
}

\begin{abstract}
RESUMEN
En este trabajo se presenta un estudio del estado del conocimiento en torno al comportamiento hidráulico de las escorias de alto horno, analizando aspectos tan variados como características e influencia de la estructura de su fase vítrea, de su composición química, mecanismos que rigen su proceso de hidratación, activadores y mecanismos del proceso de activación, etc.
\end{abstract}

Así mismo, se presentan los resultados obtenidos tras realizar una caracterización química y mineralógica de las escorias de alto horno españolas.

\section{$S U M M A R Y$}

This paper presents the state-of-the-art on hydraulic behaviour of the blast furnace slags. It analyzes different aspects such as characteristics and influence of its vitreous phase structure, its chemical composition, mechanisms that rule its hydration process, activators and activating process mechanisms, etc.

At the same time, the paper presents results obtained from a chemical and mineralogical characterization of Spanish blast furnace slags.

\section{INTRODUCCIÓN}

Las escorias de alto horno son materiales muy utilizados como adición activa para la elaboración de distintos cementos comerciales. Estos cementos siderurgicos tienen algunas propiedades y características sensiblemente mejores que los cementos portland ordinarios, tales como menor calor de hidratación, alta resistencia a los sulfatos y al agua de mar, así como una reducción muy apreciable a la reacción árido-álcalis. Sin embargo, y pese a ello existen aún muchas interrogantes en torno a estas escorias y fundamentalmente respecto a los factores ó parámetros que afectan a su comportamiento hidráulico.

En este trabajo se presenta un estudio del estado del conocimiento en torno al comportamiento hidráulico de las escorias de alto horno, analizando aspectos $\tan$ variados como características e influencia de la estructura de su fase vítrea, de su composición química, mecanismos que rigen su proceso de hidratación, activadores y mecanismos del proceso de activación, etc.
Así mismo, se presentan los resultados obtenidos tras realizar una caracterización química y mineralógica de las escorias de alto horno españolas.

\section{COMPOSICIÓN QUÍMICA Y MINERALÓGICA DE LAS ESCORIAS DE ALTO HORNO}

Las escorias siderúrgicas de alto horno son el resultado de la combinación de la ganga ácida "arcillosa" del material de hierro y de las cenizas de azufre del coque (igualmente de carácter ácido), con la cal y la magnesia (ambos compuestos básicos) de las calizas más o menos dolomíticas utilizadas como fundentes.

La combinación de los óxidos ácidos $\left(\mathrm{SiO}_{2}\right.$ y $\left.\mathrm{Al}_{2} \mathrm{O}_{3}\right)$ y óxidos básicos ( $\mathrm{CaO}$ y $\mathrm{MgO}$ ), y la formación de los constituyentes de la escoria tiene lugar por fusión a alta temperatura $\left(\approx 1.600^{\circ} \mathrm{C}\right)$, y enfriamiento del magma fluido desde $1.400^{\circ} \mathrm{C}$ hasta temperatura ambiente.

Estos subproductos industriales estan constituidos, 
tanto por fases vítreas como cristalinas. Las escorias con altos contenidos en material vítreo son de naturaleza más ácida. Existen diferentes procesos de granulación ó peletización que tienen como objetivo conseguir una escoria con una elevada proporción de fase vítrea. Una escoria granulada normal tiene un contenido en materia vítrea entre el $85-95 \%$ en peso.

La composición química de las escorias de alto horno varía entre los siguientes límites (1):

$$
\begin{array}{ll}
\mathrm{SiO}_{2} & =27-40 \% . \\
\mathrm{Al}_{2} \mathrm{O}_{3} & =5-33 \% . \\
\mathrm{CaO} & =30-50 \% . \\
\mathrm{MgO} & =1-21 \% . \\
\mathrm{Fe}_{2} \mathrm{O}_{3} & =<1 \% . \\
\mathrm{S} & =<3 \% . \\
\mathrm{Cr}_{2} \mathrm{O}_{3} & =0,003-0,007 \% . \\
\mathrm{Cl} & =0,19-0,26 \% . \\
\mathrm{TiO}_{2} & =<3 \% . \\
\mathrm{F} & =0,09-0,23 \% . \\
\mathrm{MnO}_{2} & =<2 \% . \\
\mathrm{P}_{2} \mathrm{O}_{5} & =0,02-0,09 \% . \\
\mathrm{Na}_{2} \mathrm{O}+\mathrm{K}_{2} \mathrm{O} & =1-3 \% .
\end{array}
$$

La variación en la composición química de las escorias depende del proceso siderúrgico aplicado y del tipo de acero refundido. Sin embargo, para un mismo acero, las composiciones químicas de las escorias de alto horno son bastantes similares. Los coeficientes de variación del $\mathrm{SiO}_{2}, \mathrm{CaO}$ y $\mathrm{Al}_{2} \mathrm{O}_{3}$ son inferiores al $2 \%$. Para el $\mathrm{MgO}$ alcanza valores del $6,5 \%$ y para el $\mathrm{Na}_{2} \mathrm{O}+\mathrm{K}_{2} \mathrm{O}$ está alrededor del $20 \%$. En general, las mayores desviaciones se obtienen para los elementos que están presentes como trazas.

La posición de las escorias de alto horno en el sistema ternario $\mathrm{CaO}-\mathrm{SiO}_{2}-\mathrm{Al}_{2} \mathrm{O}_{3}$ está indicada en la figura 1 (2). En este diagrama se pueden distinguir los productos ricos en cal, como los cementos portland y aluminosos, las escorias de alto horno (ya sean ácidas ó básicas), de las cenizas de lignito, de las pobres en cal situadas en la parte superior derecha como las cenizas volantes, las puzolanas, los basaltos y el humo de sílice.

Las escorias de alto horno ácidas son aquellas cuya relación $\mathrm{CaO} / \mathrm{SiO}_{2}$ es inferior a 1 , y se obtienen en procesos siderúrgicos de minerales pobres en $\mathrm{Fe}$.

En la Tabla I se resumen los minerales más importantes presentes en estas escorias (3).

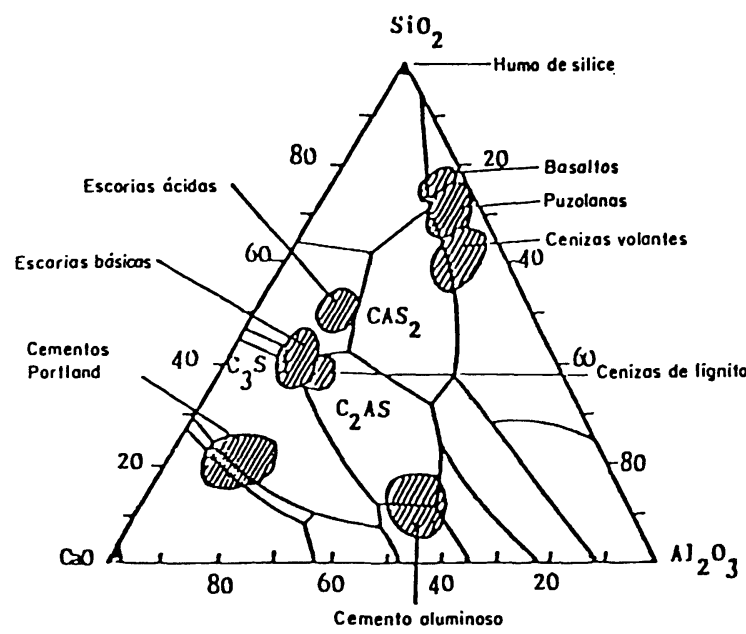

Fig. 1.-Diagrama $\mathrm{CaO}-\mathrm{Al}_{2} \mathrm{O}_{3}-\mathrm{SiO}_{2}$.

El mineral más significativo de las escorias cristalizadas es la melilita (disolución sólida de gelenita, $\mathrm{C}_{2} \mathrm{AS}\left({ }^{\star}\right)$ y akermanita $\mathrm{C}_{2} \mathrm{MS}_{2}$ ).

Minato (4) estudió mediante microscopía óptica y electrónica, el proceso de crecimiento, la estructura y composición química de los cristales de melitita en el seno de un fundido de escoria, concluyendo que la melilita cristaliza en forma dendrítica, dando lugar a unos cristales de composición química heterogénea desde el interior a los bordes. Las zonas centrales de los cristales son más ricas en gelenita, mientras que las áreas intermedias y externas tienen composiciones más próximas a las de la akermanita.

Como se desprende de la Tabla I, las escorias básicas están constituidas principalmente de melilita y merwinita $\left(\mathrm{C}_{3} \mathrm{MS}_{2}\right)$ mientras que las ácidas están formadas de melilita y diópsido.

Contrariamente a otras escorias y al clínker del cemento portland, las escorias de alto horno no tienen nunca óxidos libres, como $\mathrm{FeO}, \mathrm{CaO}$ ó MgO. Tampoco aparecen los minerales ricos en cal del clínker, tales como $\mathrm{C}_{3} \mathrm{~A}$ y $\mathrm{C}_{3} \mathrm{~S}$.

\subsection{Escorias de alto horno españolas}

F. Puertas (5) realizó una caracterización química y mineralógica de las escorias de alto horno españolas. Las escorias procedian de los Altos Hornos de Veriña y Avilés (ENSIDESA) y Vizcaya, que son las únicas factorias en España que trabajan a

(*) Nomenclatura de la Química del Cemento: $\mathrm{S}=\mathrm{SiO}_{2} ; \mathrm{C}=\mathrm{CaO} ; \mathrm{S}=\mathrm{SO}_{3} ; \mathrm{M}=\mathrm{MgO} ; \mathrm{A}=\mathrm{Al}_{2} \mathrm{O}_{3}$; $\mathrm{F}=\mathrm{Fe}_{2} \mathrm{O}_{3} ; \mathrm{H}=\mathrm{H}_{2} \mathrm{O}$. 
régimen constante. Las escorias analizadas se encontraban tanto on forma cristalizada como granulada.

Los análisis químicos de dichas escorias aparecen recogidos en las Tablas II y III, que corresponden, respectivamente, a los análisis de las escorias granuladas y cristalizas. Las mayores diferencias en composición química en las escorias granuladas de Veriña y Vizcaya residen en los contenidos en $\mathrm{SiO}_{2}$, $\mathrm{CaO}$ y $\mathrm{MgO}$. Los dos primeros son superiores en la escoria de Veriña, mientras que el MgO tiene mayores porcentajes en la de Vizcaya.

Las escorias cristalizadas de Veriña y Avilés tienen análisis químicos muy parecidos (Tabla III), presentando estas, logicamente, las mismas diferencias en composición química ya descritas, para las escorias granuladas.

El análisis mineralógico realizado a todas estas escorias se llevó a cabo a través de Difracción de Rayos X (DRX), espectroscopía de absorción infrarroja y microscopía de luz reflejada.

Desde el punto de vista mineralógico las escorias granuladas son amorfas (observado por DRX), aunque la escoria de Vizcaya presenta una pequeña proporción de fase cristalizada que ha sido identificada por DRX y corresponde a la gelenita $\left(C_{2} A S\right)$.

Por otra parte, el análisis mineralógico realizado a las escorias cristalizadas puso en evidencia que estas escorias contenian una única fase cristalina, la melilita, que corresponde como ya se ha indicado a una disolución sólida en la línea gelenita-akermanita.

Por DRX no fué posible inferir una diferenciación clara en la composición de dicha fase melilítica ya que todas presentaban unos difractogramas prácticamente idénticos; únicamente resaltar el mayor grado de cristalización observado en la escoria de Vizcaya.

A través de espectroscopía IR sí fué posible establecer algunas diferencias significativas. En la figura 2 aparecen los espectros IR, en el rango de $1.500-200 \mathrm{~cm}^{-1}$ de las tres escorias cristalizadas estudiadas. Los tres espectros IR presentan bandas anchas y difusas, con absorciones características situadas en $590,858,940,985$ y $1.030 \mathrm{~cm}^{-1}$ que confirmaban la existencia de una disolución sólida gelenita-akermanita (melilita). La absorción situada hacia $600 \mathrm{~cm}^{-1}$ corresponde a la vibración de los grupos formadores de red $\mathrm{MgO}_{4}$. Esta banda no aparecía en el caso del espectro IR de la escoria de Avilés y era de muy baja intensidad en el de Veriña; sin embargo, en el espectro de la escoria de Vizcaya sí aparece a $600 \mathrm{~cm}^{-1}$ una absorción de notable intensidad.

A partir de la interpretación de estos espectros de acuerdo a la teoría sobre "vibraciones separadas" de Tarte (6) se concluyó que la escoria de Altos Hornos de Vizcaya tenía una composición próxima a $A_{7} G_{3}$, mientras que las escorias de Veriña y Avilés la tenian próxima a $A_{5} G_{5}$, donde $A=A k e r m a-$ nita y $\mathbf{G}=$ Gelenita.

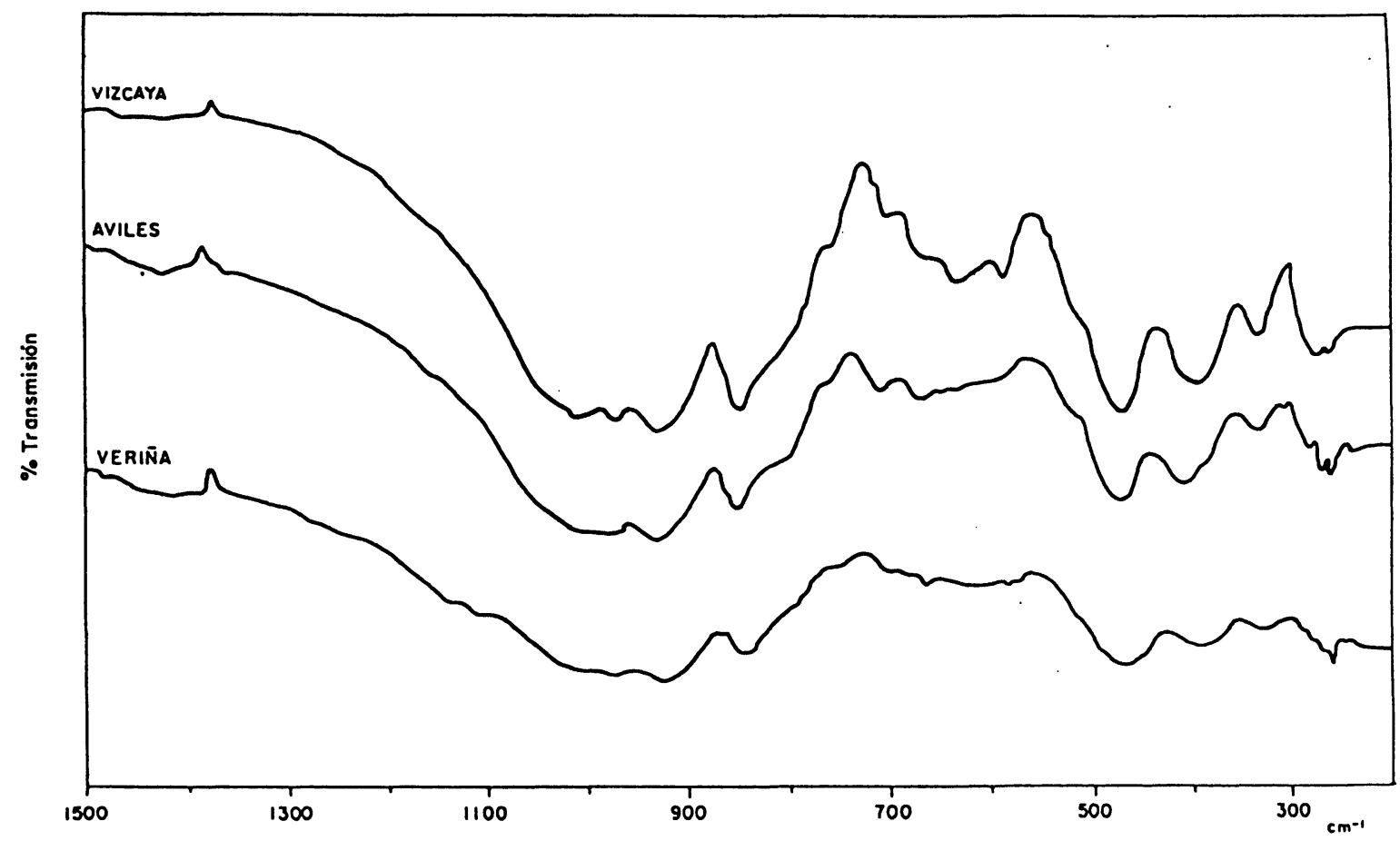

Fig. 2.-I.R. de las escorias cristalizadas. 
TABLA 1

\begin{tabular}{|c|c|c|c|c|c|}
\hline \multicolumn{2}{|c|}{ Minerales principales } & \multicolumn{2}{|c|}{ Minerales minoritarios } & \multicolumn{2}{|c|}{$\begin{array}{c}\text { Minerales minoritario raramente } \\
\text { observados }\end{array}$} \\
\hline Solución sólida & $\begin{array}{l}2 \mathrm{CaO} \cdot \mathrm{Al}_{2} \mathrm{O}_{3} \cdot \mathrm{SiO}_{2} \\
\text { (gelenita) }\end{array}$ & $\begin{array}{l}\text { Silicato bicálci- } \\
\operatorname{co}^{* *}\left(\alpha, \alpha^{\prime}, \beta\right)\end{array}$ & $2 \mathrm{CaO} . \mathrm{SiO}_{2}$ & Anortita** & $\mathrm{CaO} \cdot \mathrm{Al}_{2} \mathrm{O}_{3} \cdot 2 \mathrm{SiO}_{2}$ \\
\hline Melilita & $\begin{array}{l}2 \mathrm{CaO} \cdot \mathrm{MgO} .2 \mathrm{SiO}_{2} \\
\text { (akermanita) }\end{array}$ & Monticellita* & $\mathrm{CaO} \cdot \mathrm{MgO} \cdot \mathrm{SiO}_{2}$ & Forsterita & $2 \mathrm{MgO} . \mathrm{SiO}_{2}$ \\
\hline Merwinita* & $3 \mathrm{CaO} \cdot \mathrm{MgO} .2 \mathrm{SiO}_{2}$ & Rankinita & $3 \mathrm{CaO} .2 \mathrm{SiO}_{2}$ & Enstatita** & $\mathrm{MgO} \mathrm{SiO}_{2}$ \\
\hline Diopsido** & $\mathrm{CaO} \cdot \mathrm{MgO} .2 \mathrm{SiO}_{2}$ & $\begin{array}{l}\text { Pseudo-wollasto- } \\
\text { nita }\end{array}$ & $\mathrm{CaO}_{\mathrm{SiO}}$ & Perowskita & $\mathrm{CaO} \cdot \mathrm{TiO}_{2}$ \\
\hline Otros pyroxenos & & Oldhamita & Cas & Spinela & $\mathrm{MgO} . \mathrm{Al}_{2} \mathrm{O}_{3}$ \\
\hline
\end{tabular}

* Sólo observado en las escorias básicas.

** Sólo observado en las escorias ácidas.

TABLA \|

Análisis químicos de las escorias granuladas procedentes de los Altos Hornos de Ensidesa y Vizcaya

\begin{tabular}{|l|c|c|c|c|c|c|c|c|c|}
\hline $\begin{array}{c}\text { Altos } \\
\text { Hornos }\end{array}$ & $\begin{array}{c}\mathrm{H}_{2} \mathrm{O} \\
\text { comb. }\end{array}$ & $\mathrm{CO}_{2}$ & $\mathrm{RI}$ & $\mathrm{SiO}_{2}$ & $\mathrm{Al}_{2} \mathrm{O}_{3}$ & $\mathrm{Fe}_{2} \mathrm{O}_{3}$ & $\mathrm{CaO}$ & $\mathrm{MgO}$ & $\mathrm{SO}_{3}$ \\
\hline $\begin{array}{l}\text { Veriña } \\
\text { (Ensidesa) }\end{array}$ & 0,01 & 0,81 & 0,43 & 40,62 & 10,05 & 0,50 & 38,34 & 8,75 & 0,00 \\
\hline Vizcaya & 1,05 & 1,42 & 0,29 & 38,75 & 9,65 & 1,20 & 36,70 & 11,41 & 2,33 \\
\hline
\end{tabular}

TABLA III

Análisis químicos de las escorias cristalizadas procedentes de los Altos Hornos de Ensidesa y Vizcaya

\begin{tabular}{|l|c|c|c|c|c|c|c|c|c|}
\hline \multicolumn{1}{|c|}{$\begin{array}{c}\text { Altos } \\
\text { Hornos }\end{array}$} & $\begin{array}{c}\mathrm{H}_{2} \mathrm{O} \\
\mathrm{comb}\end{array}$ & $\mathrm{CO}_{2}$ & $\mathrm{RI}$ & $\mathrm{SiO}_{2}$ & $\mathrm{Al}_{2} \mathrm{O}_{3}$ & $\mathrm{Fe}_{2} \mathrm{O}_{3}$ & $\mathrm{CaO}$ & $\mathrm{MgO}$ & $\mathrm{SO}_{3}$ \\
\hline $\begin{array}{l}\text { Veriña } \\
\text { (Ensidesa) }\end{array}$ & 0,14 & 0,40 & 0,39 & 39,37 & 12,57 & 0,31 & 37,57 & 8,14 & 1,21 \\
\hline $\begin{array}{l}\text { Avilés } \\
\text { (Ensidesa) }\end{array}$ & 0,32 & 0,10 & 0,13 & 39,17 & 11,68 & 2,25 & 38,03 & 7,16 & 1,92 \\
\hline Vizcaya & 0,16 & 0,65 & 0,32 & 37,66 & 10,04 & 0,37 & 40,69 & 10,90 & 2,26 \\
\hline
\end{tabular}




\section{COMPORTAMIENTO HIDRÁULICO DE LAS ESCORIAS DE ALTO HORNO: INFLUENCIA DE LA ESTRUCTURA Y COMPOSICIÓN}

Las escorias de alto horno; y más concretamente las granuladas o peletizadas, tienen capacidad hidráulica latente o potencial; es decir, que finamente molidas y amasadas con agua son capaces de fraguar y endurecer. Esta capacidad hidráulica potencial de las escorias está muy atenuada y se manifiesta con lentitud, precisando de ciertos activadores para acelerar sus reacciones de hidratación.

Los parámetros que influyen sobre el comportamiento hidráulico de la escoria son: el contenido en fase vítrea, la composición química, la finura y los métodos y/o sustancias de activación.

La relación entre composición, estructura y actividad hidráulica de las escorias han sido ampliamente estudiadas por Yuan Runzhang y Col. $(7,8,9)$. Los resultados obtenidos han demostrado que dicha actividad hidráulica depende principalmente de su estructura; y que la estructura de la escoria está íntimamente relacionada con su composición química y su historia térmica.

El primer parámetro estructural a caracterizar es la relación de los contenidos en fase vítrea y cristalina. Como es conocido, en la fase vitrea reside el componente hidráulicamente activo de la escoria, pudiendo ser considerada la fase cristalina prácticamente como un inerte.

Al enfriar bruscamente la escoria líquida (procesos de granulación, peletización, etc.) se obtiene un vidrio de forma irregular y gran energía interna (aproximadamente el calor de cristalización es de $200 \mathrm{~J} / \mathrm{g}$ ), lo que hace que sea un material altamente inestable y de gran reactividad.

Los contenidos tanto en fase vítrea como cristalina en una escoria se suelen determinar por análisis cuantitativo por Difracción de Rayos X (DRX) (10) y por microscopía óptica. Hooton y Emery (11) utilizaron la microscopía óptica de luz transmitida con una lámina de yeso que coloreaba a las fases vítreas y cristalinas de rosa y amarillo o azul, respectivamente. Un recuento de 150 partículas con un tamaño de grano comprendido entre 45-63 $\mu \mathrm{m}$, da resultados concordantes con los obtenidos por DRX.

Frearson y Col. (12) emplean microscopía óptica de reflexión. El ataque a las superficies pulidas lo hacen con una disolución de ácido nítrico en etanol. En este caso el vidrio tiene color marrón y los cristales de merwinita toman una coloración azulada y los de melilita permanecen incoloros. El recuento se realiza sobre 500 puntos.

\subsection{Estructura de la escoria vítrea de alto horno}

De acuerdo a la teoria de Zachariasen (13), un vidrio es una red tridimensional, más o menos deformada, de átomos formadores de red que están rodeados de 4 átomos de oxígeno formando tetraedros, de manera que cada átomo de oxígeno forma parte de dos tetraedros.

El Si es el típico formador de red de los vídrios. En las escorias se puede presentar como tetraedros $\mathrm{SiO}_{4}{ }^{4-}$, grupos $\mathrm{Si}_{2} \mathrm{O}_{7}{ }^{6-}$ o cadenas de otros polímeros, tales como $\mathrm{Si}_{3} \mathrm{O}_{9}^{6-}, \alpha$-wollastonita o B-wollastonita $\left(\mathrm{SiO}_{3}\right)_{\mathrm{n}}(14)$. Las valencias negativas de estos grupos aniónicos están neutralizadas por las valencias positivas de los cationes, llamados modificadores de red. El ion $\mathrm{Ca}^{2+}$ es un típico modificador de red y está coordinado octaédricamente.

Dron (15) considera la fase vítrea de la escoria basada en una red macroaniónica de aluminosilicato, en la que el aluminio ocupa los nódulos de la red y los extremos están ocupados por cadenas de silicato, tal y como puede visualizarse en la figura 3.

El grado de polimerización de los tetraedros $\mathrm{SiO}_{4}{ }^{4-}$ disminuye al aumentar la proporción de modificadores de red, lo cual quiere decir que a mayor cantidad de éstos mayor desorden estructural $y$, por tanto, mayor reactividad.

$\mathrm{Al}_{2} \mathrm{O}_{3}$ y $\mathrm{MgO}$ son óxidos anfóteros, es decir, se pueden encontrar como $\mathrm{AlO}_{4}{ }^{5-}$ y $\mathrm{MgO}_{4}^{6-}$, y actuar como formadores de red, 0 como $\mathrm{Al}^{3+}$ y $\mathrm{Mg}^{2+}$ (octaédricamente coordinados) y actuar como modificadores de red. Si el Mg y el Al actúan como modificadores de red y/o la cantidad de $\mathrm{Ca}^{2+}$ es

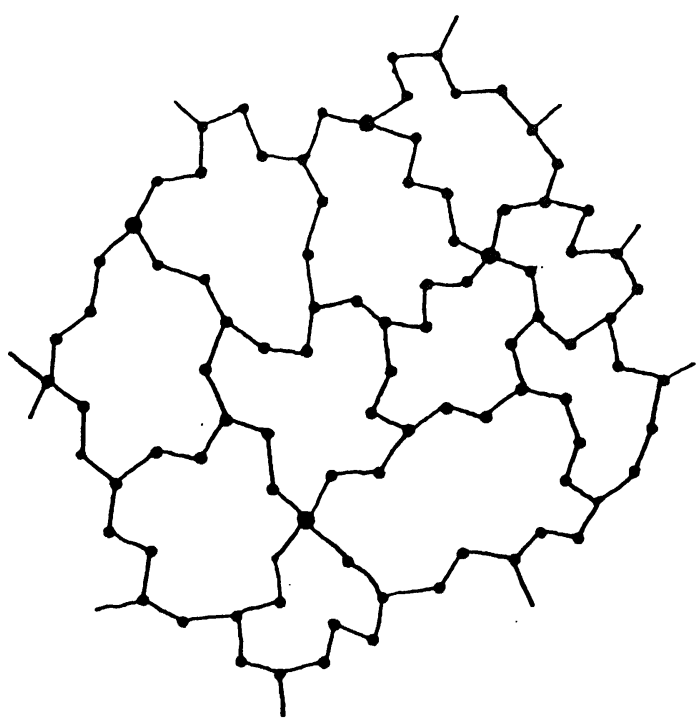

Fig. 3.-Estructura esquemática de la redmacronónica de alumino-silicato. 
elevada, se impide la polimerización de los tetraedros $\mathrm{SiO}_{4}^{4-}$, lo que hace que el sistema sea menos estable y aumente notablemente su reactividad hidráulica (16).

Otros autores (17) indican que cuando el Al actúa como formador de red (coordinación 4) es altamente eficaz, desde el punto de vista hidráulico, ya que los tetraedros $\mathrm{AlO}_{4}^{5-}$ tienen uniones más débiles que los tetraedros $\mathrm{SiO}_{4}{ }^{4-}$, y por lo tanto son más fáciles de disolver.

La siguiente relación establecida por Satarín (18) parece ser la que proporciona la óptima reactividad:

$$
\frac{\mathrm{MeO}_{6}}{\mathrm{MeO}_{4}}=0.35, \text { donde } \mathrm{Me}=\mathrm{Al} \text { y } \mathrm{Mg}
$$

La opinión generalizada es que cuanto más desordenada es la estructura del vidrio mayor es su hidraulicidad.

Hay otros autores (3) que consideran que la presencia de una pequeña proporción de fase cristalizada puede contribuir a un incremento en la reactividad de la escoria; pues es conocido que el inicio de la cristalización produce una estructura con mayor desorden. Las mayores resistencias se alcanzan cuando los constituyentes cristalinos están distribuidos homogéneamente en la fase vítrea, en un estado de núcleos submicroscópicos. Demoulian (19) encontró que un $5 \%$ de fase cristalina incrementaba las resistencias a 2 y 28 días debido a un fenómeno de nucleación durante el proceso de hidratación. Según Sersale (20), una cantidad moderada de fracción cristalina en la escoria (alrededor de un 3-5\% en peso), puede ser considerada como micropartículas de escoria no reactiva que actuan como barreras que impiden la propagación de las microfisuras.

Aun cuando la reactividad de la escoria se reduce cuando aumenta la desvitrificación, sin embargo la relación entre el contenido de vidrio en la escoria y la resistencia desarrollada por cementos de escorias no es lineal (21).

Las escorias de alto horno utilizadas para la elaboración de cementos con escoria deben tener unos contenidos muy elevados en fase vítrea, del orden del $95 \%$ o superiores.

El contenido de vidrio para la hidraulicidad potencial de la escoria siderúrgica es, según Calleja (22), más importante que la composición química de la misma; ya que ha observado que escorias con índices de hidraulicidad mediocres dan excelentes resultados, con tal de que tengan porcentajes de fase vítrea elevados.

\subsection{Influencia de la composición química}

De entre las escorias de alto horno, aquellas que tienen mayor potencial hidráulico son las básicas y de naturaleza vítrea.

Hay diferentes índices y módulos que pretenden establecer la hidraulicidad y basicidad óptimas de las escorias respecto a su comportamiento hidráulico.

Los índices de basicidad $p$ de las escorias tienen las expresiones:

$$
P_{1}=\frac{C}{S} \quad P_{2}=\frac{C+M}{S} \quad P_{3}=\frac{C+M}{S+A}
$$

Los índices de hidraulicidad clásicos estan reflejados en la Tabla IV. El primer grupo de $F_{1}$ a $F_{7}$ relaciona los constituyentes mayoritarios de la escoria. De todos ellos, $F_{3}$ es el más conocido y aceptado en varios paises, cuyo valor tiene que ser mayor o igual a $1-1,4$.

En los módulos $F_{8}$ a $F_{10}$, los elementos minoritarios como el manganeso son tenidos en cuenta. En el caso de los índices $F_{11}$ y $F_{12}$ la hidraulicidad de la escoria disminuye para contenidos crecientes de $\mathrm{Al}_{2} \mathrm{O}_{3}$

Se han calculado las correlaciones entre los distintos índices hidráulicos y las resistencias a compresión a 2 y 28 días obtenidas en distintos cementos de escoria (3). Los resultados han demostrado que los índices del $F_{1}$ al $F_{9}$ son los que dan las mejores correlaciones.

Kondo (23) indicó que vidrios de escoria con contenidos superiores al $50 \%$ en $\mathrm{CaO} \theta$ inferiores al $20 \%$ en $\mathrm{SiO}_{2}$ tienen propiedades hidráulicas. En general, y en esto sí hay acuerdo entre los autores, incrementos en el contenido de $\mathrm{SiO}_{2}$ tiene efectos negativos en cuanto a la hidraulicidad, mientras que incrementos en el contenido de $\mathrm{CaO}$ tiene efectos positivos.

El papel jugado por el $\mathrm{Al}_{2} \mathrm{O}_{3}$ y $\mathrm{MgO}$ es objeto de mayores contradicciones. Para algunos autores (24) la presencia de $\mathrm{Al}_{2} \mathrm{O}_{3}$ en contenidos hasta un $21 \%$ es muy positiva; para otros con porcentajes muy superiores al $10 \%$ produce una disminución de resistencias a 7 y 28 dias.

Respecto al MgO, Smolczyk (3) dice que hasta un $11 \%$ juega un papel comparable al del $\mathrm{CaO}$. Otros autores (25) indican un papel nefasto del $\mathrm{MgO}$; otros correlacionan los contenidos de $\mathrm{MgO}$ con los de $\mathrm{Al}_{2} \mathrm{O}_{3}$ para una reactividad óptima (26). En definitiva, no está claro cuáles deben de ser los 
TABLA IV

Índices de hidraulicidad de las escorias

\begin{tabular}{|lrr|}
\hline$F_{1}=100-S$ & $F_{2}=\frac{100-S}{S}$ & $F_{3}=\frac{C+M+A}{S}$ \\
$F_{4}=\frac{C+M+A-10}{S+10}$ & $F_{5}=\frac{C+1,4 M+0,64 A}{S}$ \\
$F_{6}=C+0,5 M+A-2,05$ & $F_{7}=\frac{6 C+3 A}{7 S+4 M}$ \\
\hline$F_{6}=\frac{C+0,5 M+A+C a S}{S+M n O}$ & $F_{8}=\frac{C+0,5 M+A}{S+F \theta+(M n O)^{2}}$ \\
\hline$F_{11}=\frac{C+M+0,3 A}{S+0,7 \mathrm{~A}}$ & $F_{10}=\frac{C+M+A+B a O}{S+M n O}$ & $F_{12}=\frac{C+M}{S+0,5 \mathrm{~A}}$ \\
\hline
\end{tabular}

contenidos óptimos ni el papel real que juegan respecto a la hidraulicidad.

Para la mayoría de los autores, el $\mathrm{MnO}$ tiene un efecto negativo, provocando un descenso en las resistencias (27). Sin embargo, se han observado excelentes propiedades hidráulicas en escorias de alto horno con un $14 \%$ en $\mathrm{MnO}$ (28). La presencia de álcalis y $\mathrm{P}_{2} \mathrm{O}_{5}$ en la escoria parece tener un efecto complejo y no del todo conocido sobre la actividad hidráulica de dicha escoria (29). $\mathrm{EI} \mathrm{TiO}_{2}$ disminuye las resistencias cuando se encuentra en contenidos superiores al $4 \%$. Este efecto negativo parece ser atenuado adicionando álcalis al clínker (30).

Finalmente, indicar que Totani y Col. (31) han estudiado el comportamiento hidráulico de escorias de igual composición y contenido en fase vítrea. Los resultados obtenidos han demostrado que el comportamiento de estas escorias era, pese a todo, diferente. Parece que el aspecto más importante, y sobre el que debería incidirse en futuras investigaciones, es el conocimiento más profundo de la estructura del vidrio y la relación de esta con la reactividad de dicha escoria. En este sentido, Rojak, Shkolnik y Orlow (32) dicen que la actividad de las escorias es función del grado de despolimerización de los complejos Si-O y de la energía del enlace Me-O del catión despolimerizado, y de la coordinación de este último. Estos factores pueden diferenciar escorias de igual composición química y diferente hidraulicidad.
Las escorias cristalizadas de alto horno tienen baja actividad hidráulica, aunque también ésta varía dependiendo de la composición mineralógica de las fases microcristalinas de dicha escoria. Yan RunZang y Col. (7) encontraron que la hidraulicidad disminuía cuando la proporción de esa fase microcristalina en la escoria iba en el siguiente orden: $\mathrm{C}_{2} \mathrm{~S}>\mathrm{C}_{2} \mathrm{AS}>\mathrm{C}_{3} \mathrm{~S}_{2}>\mathrm{CS}$.

\subsection{Mecanismos de hidratación de las escorias de alto horno}

La hidratación de la escoria granulada resulta de su disolución en la fase acuosa y en la recombinación en el seno de esta última de los iones liberados. En la fase inicial, en la que no se han producido aún las primeras precipitaciones de C-S-H, son los elementos estructurales más básicos del vidrio, los que pasan preferentemente a la solución; es decir, las entidades $\left(\mathrm{SiO}_{3.5}\right)^{3-}$ ó $1 / 2\left(\mathrm{Si}_{2} \mathrm{O}_{7}\right)^{6-}$ y $\mathrm{AlO}_{2}^{-}$. Esta disolución es hidrolítica y no hidroxílica. Esto explica la reacción básica de la escoria (hay que indicar que estas mismas entidades se encuentran en los retículos cristalinos de la rankinita, $C_{3} S_{2}, y$ CA).

La disolución es de tipo congruente, y la relación C/S del C-S-H es inferior a la de la escoria, por lo que la disolución se enriquece en $\mathrm{Ca}$, y posteriormente se empobrece en Si. Además, el Al se acumula en la disolución hasta la cristalización que se produce posteriormente a la precipitación inicial del C-S-H. 
Recientemente, Glasser (33) ha propuesto un mecanismo de hidratación de las escorias vítreas de alto horno en una matriz de pasta de cemento portland. En la figura 4 se muestra la evolución de dicho proceso. Inicialmente, estos granos de escoria sufren un ataque químico debido al elevado $\mathrm{PH}$ de la fase líquida en contacto con ellos.

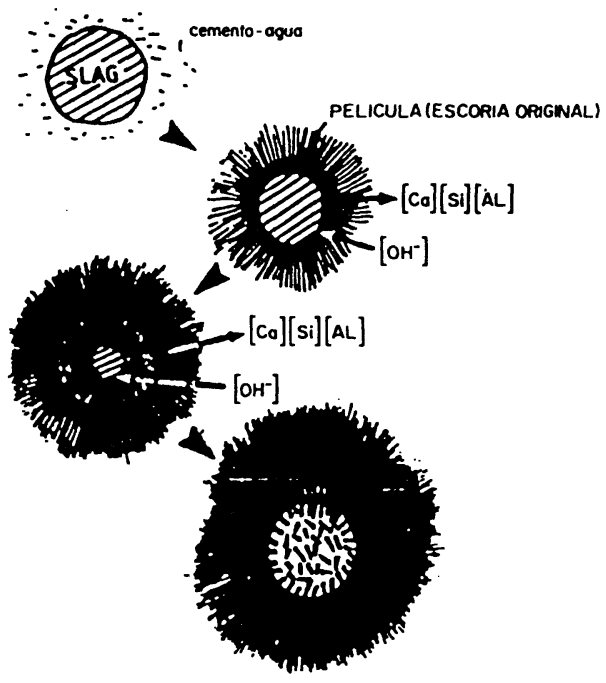

Fig. 4.-Representación esquemática de la hidratación de la escoria.

Como consecuencia de dicho ataque se forman productos de hidratación muy insolubles en la superficie del grano, creando una capa semiprotectora que actúa como barrera impidiendo que las reacciones prosigan a una velocidad conveniente. Estos productos de hidratación son amorfos a la difracción de rayos $X$ y a la difracción de electrones, ya que son geles tipo C-S-H con relaciones $\mathrm{C} / \mathrm{S}$ inferiores a las presentes en las pastas de cemento portland. Esta capa aisla parcialmente la parte más anhidra de los granos de la escoria.

Esta situación es inestable ya que se desarrollan diferentes potenciales químicos entre las partículas de escoria y la matriz de pasta de cemento, que conducen a que la reacción de hidratación prosiga.

I. Teoreanu (14) propone un mecanismo de acuerdo al siguiente modelo:

$$
\begin{aligned}
& -S_{1}^{1} i-\mathrm{O}-S_{1}^{1} i-+\mathrm{HO}^{-} \rightarrow-S_{1}^{1} i-\mathrm{O}-+ \\
& +-S_{1}^{\prime} i-O H \\
& -S_{1}^{\prime} i-\mathrm{O}-\stackrel{\cdot}{ }+\mathrm{Ca}^{2}+\rightarrow-S_{1}^{\prime} i-\mathrm{O}-\mathrm{Ca}- \\
& -S_{1}^{\prime} i-\mathrm{O}-\mathrm{Ca}+\mathrm{HO} \rightarrow-S_{1}^{1} i-\mathrm{O}-\mathrm{Ca}-\mathrm{OH} \\
& -S_{1}^{1} i-\mathrm{O}-\mathrm{Ca}-\mathrm{OH}+2\left(\mathrm{HO}-S_{1}^{1} i-\right) \rightarrow \\
& -S_{1}^{\mathfrak{S} i}-\mathrm{O}-\mathrm{S}_{1}^{\mathrm{S}} \mathrm{i}-\mathrm{OH}+\mathrm{Ca}(\mathrm{OH})_{2}
\end{aligned}
$$

La presencia de altas concentraciones de grupos $\mathrm{OH}^{-}$provoca el ataque hidroxílico a los grupos o enlaces siloxanos de la red macroaniónica de la escoria, destruyendo dichos enlaces por policondensación. Los grupos $\left(\mathrm{SiO}_{4}\right)$ así formados son capaces de unirse a los iones $\mathrm{Ca}^{2+}$ para dar lugar unos compuestos hidrosilicatos de calcio.

También se ha sugerido el ataque hidrolítico de los enlaces siloxanos a elevadas temperaturas, a través del siguiente proceso (14):

$$
-\stackrel{1}{S}_{1} i-O-S_{1}^{1} i-+H O H \rightarrow 2\left(-S_{1}^{1} i-O H\right)
$$

Estos mecanismos se pueden justificar en base a los dos puntos de vista siguientes (14):

(i) Por ataque hidroxílico-hidrolítico y disolución congruente de la escoria, se conduce, cuando se alcanzan las condiciones de sobresaturación en la disolución, a la precipitación de un producto hidratado que evoluciona con el tiempo. Este mecanismo se adapta mejor a las escorias de naturaleza ácida que aquéllas situadas en la línea recta CS-CA del sistema ternario $\mathrm{CaO}$ $\mathrm{Al}_{2} \mathrm{O}_{3}-\mathrm{SiO}_{2}$.

(ii) Por ataque hidroxílico-hidrolítico y disolución incongruente de los constituyentes estructurales de la escoria, con la formación de productos de naturaleza gel con estructura de hidrosilicatos de cálcio (C-S-H), tal mecanismo es más plausible que en el caso de las escorias de carácter más básico.

\subsection{Mecanismos de activación de las escorias}

Como se desprende de lo anteriormente expresado, la hidratación de las escorias requiere la ruptura de los enlaces y la disolución de la red tridimensional de alumino-silicatos de la misma. Este proceso se puede ver favorecido y acelerado por la presencia, durante el proceso de hidratación de dicha escoria, de distintos activadores.

En general, se puede decir que el grado de hidraulicidad de la escoria depende de la cantidad y composición del vidrio y del activador. Así, mientras que las escorias de vidrio de composición akermanita se pueden hidratar, de manera conveniente, sin precisar casi activador, los vidrios de composición gelenita precisan de activador.

La activación de las escorias puede realizarse a través de las siguientes vías:

(i) Activación Química 
(ii) Activación Mecánica.

\section{(iii) Activación Térmica.}

De las tres, es la activación química la más conocida y utilizada. Esta activación puede ser de naturaleza alcalina (a través de la acción del $\mathrm{Ca}(\mathrm{OH})_{2}$, $\mathrm{NaOH}, \mathrm{KOH}, \mathrm{Na}_{2} \mathrm{CO}_{3}$, silicatos de sodio, etc.), ya que a valores altos de $\mathrm{PH}$ los enlaces $\mathrm{Si}-\mathrm{O}$ y Al-O de la estructura vítrea de la escoria se rompen con facilidad, dando lugar a la precipitación de productos de baja solubilidad, tales como silicatos cálcicos hidratados, aluminatos de calcio o de magnesio hidratados, etc. También es posible esta activación con sales de naturaleza neutra, tales como $\mathrm{Na}_{2} \mathrm{SO}_{4}$, $\mathrm{CaSO}_{4}, \mathrm{NaCl}$, yeso, etc.

El $\mathrm{Ca}(\mathrm{OH})_{2}$ es un buen activador químico de las escorias. El $\mathrm{Ca}(\mathrm{OH})_{2}$ que se forma como consecuencia de la hidratación del cemento portland puede ser utilizado en la activación de las dichas escorias. En este fundamento se basan los cementos con adiciones de escorias (cementos II/S) y los cementos de horno alto (cementos III).

Teoreanu (14) propone un mecanismo de la hidratación en el sistema cemento portland-escoria-agua. Este mecanismo ocurre a través de las siguientes etapas:

a) Inmediatamente después del amasado con el agua tiene lugar una rápida evolución de calor debido a la hidratación del cemento e incluso de la escoria. Este periodo se considera como un periodo de preinducción, a través del cual el yeso aportado por el cemento portland es el principal activador de la hidratación de la escoria.

b) Se forman los primeros productos de hidratación, los cuales son de muy baja cristalinidad. El periodo de inducción empieza aproximadamente a las 12 horas de hidratación. Se inicia una acumulación de $\mathrm{Ca}(\mathrm{OH})_{2}$ como resultado de la hidrólisis del cemento.

c) Después de, aproximadamente, las primeras 12 horas de hidratación el proceso se acelera, iniciándose el primer periodo de aceleración, que se prolonga hasta aproximadamente los tres primeros días de hidratación. $\mathrm{El} \mathrm{Ca}(\mathrm{OH})_{2}$ comienza a ser el principal agente activante de la interacción entre la escoria y el agua. La cantidad de $\mathrm{Ca}(\mathrm{OH})_{2}$ en el sistema cae.

d) Se produce, entonces, una disminución en la velocidad de hidratación de la escoria, hasta que aumenta nuevamente la cantidad de hidróxido cálcico en el sistema. e) Cuando la concentración de $\mathrm{Ca}(\mathrm{OH})_{2}$ vuelve a aumentar, la hidratación de la escoria se acelera nuevamente, iniciándose el segundo periodo de aceleración más intenso, que se prolonga hasta los 28 días de hidratación. Durante este periodo, los compuestos hidratados resultantes se encuentran junto a las partículas de cemento, mientras que aquéllos formados a partir de la escoria anhidra están depositados en los poros del sistema.

f) Finalmente, y especialmente cuando los espacios disponibles entre los granos es limitado, la velocidad de hidratación del sistema está controlada por los fenómenos de difusión a través de los productos de hidratación a la interfase de la escoria que aún permanece anhidra (período de difusión final).

Mencionar, unicamente, que el inicio y duración de cada etapa cinética en la interacción de la escoria con el agua, influye no sólo la naturaleza del activador sino también del tamaño de grano y composición de la mezcla reaccionante.

Kondo y Col. (34) estudiaron el efecto del $\mathrm{Ca}(\mathrm{OH})_{2}$ en la hidratación de cementos supersulfatados de escorias. Como conclusiones de sus trabajos indican que en ausencia de $\mathrm{Ca}(\mathrm{OH})_{2}$ se forma gel C-S-H y se libera $\mathrm{Al}_{2} \mathrm{O}_{3}$ de la escoria. La formación de ettringita se detecta por SEM después de un día de hidratación. En presencia de $\mathrm{Ca}(\mathrm{OH})_{2}$ se observa una aceleración de las reacciones iniciales con formación de ettringita a las 5 horas de hidratación.

Al progresar estas reacciones se forma una capa superficial de ettringita alrededor de los granos de escoria, aún anhidros, que inhibe temporalmente la posterior hidratación de dicha escoria.

En la activación por yeso, la concentración de Ca y Al se reduce en la fase líquida, permitiendo la formación de ettringita. Cuando el pH de esa fase líquida alcanza valores próximos a 12, la ettringita se vuelve estable (9) y la hidratación de la escoria pregresa a mayor velocidad. Con el objeto de mantener activa la hidratación de esta escoria es preciso suministrar la cantidad suficiente de hidroxilos, para asegurar altos valores de PH para que continue la rúptura de los enlaces de la estructura de la escoria, y estabilizar la ettringita formada; junto con los contenidos adecuados de $\mathrm{SO}_{3}$ y alúmina precisos para la formación de la citada ettringita. El yeso acelera las reacciones de hidratación de la escoria, pero es menos efectivo como activador en ausencia de $\mathrm{Ca}(\mathrm{OH})_{2}$ (20)

Recientemente, Dutta y Col. (35) han estudiado la activación de escorias indias, bajas en cal (26$38 \%)$ y ricas en alúmina (20-30\%), con anhidrita. 
Los resultados obtenidos han demostrado que la cantidad óptima de anhidrita precisa para fabricar cementos supersulfatados con esas escorias se encuentra en el rango del $15-20 \%$ en peso, siendo la ettringita y un silicato cálcico hidratado los principales productos de hidratación formados.

Los mecanismos de activación e hidratación de la escoria son distintos según los activadores. Para Kondo (23) el $\mathrm{Ca}(\mathrm{OH})_{2}$ y el yeso actúan como especies reaccionantes, mientras que el $\mathrm{NaOH}$ juega un papel de catalizador. Voinovitch y Dron (36) indican también que el $\mathrm{NaOH}$ es una sustancia catalítica en el proceso de hidratación de la escoria.

Respecto a esta última afirmación, Narang y Chopra (37) tienen otra opinión, ya que ellos creen que el $\mathrm{NaOH}$ puede actuar como reaccionante de modo comparable a la cal y yeso, debido a su fuerte influencia catiónica. Además, según estos mismos autores, el hecho de que sea cual sea el activador, el principal producto de hidratación de la escoria es el C-S-H (aunque otros productos como la ettringita también se pueden formar cuando hay yeso ó $\mathrm{C}_{2} \mathrm{ASH}_{8}$ con $\mathrm{NaOH}$ ), y que todos estos compuestos se forman a través de un mismo mecanismo de apertura de la estructura; silicato y sustitución de iones indica que el $\mathrm{NaOH}$ no puede actuar como un "simple catalizador".

Con $\mathrm{NaOH}$ las reacciones de hidratación se completan antes (28). Según Kondo (23), con $\mathrm{NaOH}$ las reacciones se aceleran a primeras edades, y luego se mantienen prácticamente constantes a 3 y 7 días. El silicato sódico y el carbonato sódico tienen un comportamiento similar al $\mathrm{NaOH}$. Recientemente, Douglas y Brandstetr (38) comprobaron que la adición de $1 \%$ de $\mathrm{Na}_{2} \mathrm{SO}_{4}$ a morteros de escoria se obtenían unas resistencias a compresión a un día comparables a las que desarrollaba un mortero de iguales características de cemento portland. Comprobaron, así mismo, que la adición de lignosulfonatos de sodio o superplastificantes basados en sulfonaftalenos no mejoraba la trabajabilidad de dichos morteros de escorias.

Voinovitch y Dron (36) han propuesto las siguientes reacciones de hidratación de la escoria por activación:

$$
\begin{aligned}
\mathrm{C}_{5} \mathrm{~S}_{3} \mathrm{~A} & +2 \mathrm{C}+16 \mathrm{H} \longrightarrow \mathrm{C}_{4} \mathrm{AH}_{13}+\mathrm{C}-\mathrm{S}-\mathrm{H} \\
\mathrm{C}_{5} \mathrm{~S}_{3} \mathrm{~A} & +2 \mathrm{CS}+76 / 3 \mathrm{H} \longrightarrow 3 \mathrm{C}-\mathrm{S}-\mathrm{H}+ \\
& +2 / 3 \mathrm{C}_{3} \mathrm{~A} \cdot 3 \mathrm{CS} \cdot \mathrm{H}_{32}+2 / 3 \mathrm{AH}_{3}
\end{aligned}
$$

Regourd (39) indica que la ctivación alcalina por $\mathrm{NaOH}$ produce un gel C-S-H con una relación $\mathrm{C} / \mathrm{S}$ inferior a la existente en el C-S-H de la pasta del clínker portland, y se forma también $\mathrm{C}_{4} \mathrm{AH}_{13}$ y $\mathrm{C}_{2} \mathrm{ASH}_{8}$. En la activación cálcica los productos hidratados compatibles con $\mathrm{Ca}(\mathrm{OH})_{2}$ son un gel C$\mathrm{S}-\mathrm{H}, \mathrm{C}_{4} \mathrm{AH}_{13}$. El $\mathrm{C}_{2} \mathrm{ASH}_{8}$ (gelenita hidratada) no se forma; esta fase sólo aparece en presencia de $\mathrm{NaOH}$ o silicato o carbonato de sodio.

La activación sulfática produce gel C-S-H, ettringita y $\mathrm{AH}_{3}$. Esta activación es más lenta; y puede ser producida a parte de por el yeso, por el hemidridrato, la anhidrita $e$ incluso por el azufre presente en la propia escoria que puede actuar como autoactivante.

La activación conjunta de yeso y $\mathrm{NaOH}$ produce $\mathrm{Na}_{2} \mathrm{SO}_{4}$. Esta activación requiere cinco veces más de activante que la activación cálcica, pero se forma el doble de productos de hidratación.

Desde el punto de vista mecánico, se ha comprobado que la adición de pequeñas cantidades de $\mathrm{NaBr}$, $\mathrm{NaF} \circ \mathrm{Na}_{2} \mathrm{SO}_{4}$, a escorias de alto horno, incrementa la resistencia a edades tempranas; el $\mathrm{NaBr}$ y el $\mathrm{NaCl}$ la aumentan también hasta edades superiores a los 91 días. El análisis microestructural de las pastas conteniendo $\mathrm{NaBr} \circ \mathrm{NaCl}$ muestran una estructura compuesta por gel C-S-H, monosulfoaluminato, ettringita y otras fases, formando todas ellas una estructura muy compacta (20).

Smith y Osborne (40) estudiaron unos nuevos cementos basados en la mezcla de escorias granuladas de alto horno (60\% en peso), cenizas volantes silicoaluminosas (40\%) y como activador el $\mathrm{NaOH}(7 \%)$. Los resultados obtenidos han demostrado que los nuevos cementos tienen unas altas resistencias que ligeramente son luego incrementadas a mayores edades. El gran incoveniente de estos nuevos cementos es su durabilidad frente a la reacción árido-álcalis y la posible formación de eflorescencias de sales alcalinas. Sin embargo, en opinión de esos mismos autores las características positivas de los nuevos cementos compensarían los problemas de durabilidad que pudieran crear.

Las escorias también se pueden activar mecánicamente. Dimitriev (41) activó mecano-químicamente cementos de escorias por molienda, hasta destruir la red de silicatos y activar los iones oxígeno. Narang y Choppa (37) encontraron que la finura óptima de la escoria debe ser del orden de 4.000$5.000 \mathrm{~m}^{2} / \mathrm{kg}$ (Blaine). Resultados experimentales (42) han demostrado que la distribución en el tamaño de las partículas del clínker afecta más a las resistencias iniciales en un cemento de escorias, mientras que la distribución en el tamaño de las partículas de la escoria influye más sobre las resistencias del cemento a edades más avanzadas (28-90 días). 
Las escorias de alto horno pueden sustituir a la mayor parte de los materiales cementicios, siempre y cuando no se requieran altas resistencias iniciales. Sin embargo, estas se pueden alcanzar reduciendo el tamaño de las partículas de escoria, aumentando la temperatura de curado o utilizando bajas relaciones agua/cemento+escoria (20)

Finalmente, mencionar la activación térmica de las escorias se puede lograr con tratamiento en autoclave, siendo el gel C-S-H el principal producto de hidratación.

\section{BIBLIOGRAFÍA}

(1) UCKIKAWA, H. VIII Congreso Internacional de la Química del Cemento (Rio de Janeiro). Vol I, Tema III, pp. 249-280 (1986)

(2) REgouRd, M. VIII Congreso Internacional de la Química del Cemento (Rio de Janeiro). Vol I, Tema III, pp. 200-229 (1986)

(3) SMOLCZYK, H.G. VII Congreso Internacional de la Química del Cemento (París), Vol I, Tema II, pp. 1-16 (1980)

(4) MINATO, H. VII Congreso Internacional de la Química del Cemento (París), Vol IV, pp. 263-269 (1980)

(5) PUERTAS, F. Tesis Doctoral. Universidad Autónoma de Madrid (1987)

(6) TARTE, P. Bull. Soc. FranÇ. Ceram. n. 58, pp. 13-33 (1963)

(7) RUN-ZANG, Y; QIONG-YING, G. Silic. Ind. n.2 12, pp. 279-281 (1982)

(8) RUN-ZANG, Y; OUYANG, S; QIONG-YING, G. Silic. Ind. n. 1,pp. 3.6 (1983)

(9) RU-ZANG, Y; QIONG-YING, G; OUYANG, S.: Silic. Ind. n. 3-4 pp. 55-59 (1988)

(10) KLUG, H. P.; ALEXANDER, L. E.: "X- ray diffraction procedures for polycrystalline and amorphous materials". John Wiley \& Sons (1954)

(11) HOOTON, R.E.; EMERY, J. J.: Ist. Intern. Conf (Montebello). Vol II, pp. $943-962$ (1983)

(12) FREARSON, J. P. J.; SIMS, I; UREN, J. M.: 2nd. Intern. Conf. Concr. (Madrid),pp. 21-25 (1986)

(13) ZACHARIASEN, W. H.: J. Am. Ceram. Soc. Vol 54, pp. $3841-3851$ (1932)

(14) TEOREANU, I.: ॥ cemento, n. 2 pp. $91-97$ (1991)

(15) DRON, R.: Silic. Indus. n. 6 , pp.143-147 (1982)

(16) ROYAK, S. M.: Tsement, n. ${ }^{2} 8$, p. 415 (1978)

(17) PORK, V.: Glassy State, Nauka, PP. 354-356 (1971)

(18) SATARIN, V. I.: VI Congreso Internacional de la Química del Cemento (Moscú) Vol I,. pp. 1-51 (1974)

(19) DEMOULIAN, E; GOURDIN, P; HAWTHORN, F: VERNET, C.: VIII Congreso Internacional de la Química del Cemento (París), Vol II, pp. III-89 (1980)

(20) SERSALE, R.: IX Congreso Internacional de la Química del Cemento (New Delhi). Vol I, Tema IIA , pp. 261-302 (1992)

(21) DOUGLAS, E.; ZERBINO, R.: Cem. Concr. Res. Vol 16, pp. $662-670$ (1986)

(22) CALLEJA, J.: Materiales de Construcción, n. ${ }^{2}$ 168, pp. 11-35 (1985)

(23) KONDO, R.; UEDA, S.: V Congreso Internacional de la Química del Cemento (Tokyo). Vol ii-4, pp.203-255 ( 1968)

(24) CHOPPA, K.; TANEJA, C.A.: V Congreso Internacional de la Quimica del Cemento (Tokyo), Vol IV. pp. 228-236 (1968)

(25) CASSELOURI, W; PARISSAKIS, G.: Silc. Ind. 42 (1), pp. 13-17 (1977)

(26) ROJAK S. M.; CHKOLNIK, J. Ch.: VII Congreso Internacional de la Química del Cemento (París). Tema III, pp. 74-77 (1980)

(27) VYLKOVA, I. S.: VI Congreso Internacional de la Quimica del Cemento (Moscú). Sección III/2 (1974)

(28) NARANG, K. C.: IX Congreso Internacional de la Química del Cemento (New Delhi), Vol I, Tema II, pp. 213-257 (1992) 
(29) ROJAK, S. M.; SHKOLNIK, Y. S.; OKONISKY, N. H.; SLEPTSOV, Z. E.; ADMAKIN, F. K.; FEDOROVA,T. M.: Stroitelrye

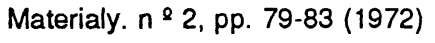

(30) GovoroV, A. A.: VIII Congreso Internacional de la Química del Cemento (Rio de Janeiro), Vol IV, pp.128 (1986)

(31) TOTANI, Y; SAITO, Y; NAGEYARIA, Y; TANAKA, M.: VIII Congreso Internacional de la Química del Cemento (Rio de Janeiro). Tema III (1986)

(32) ROJAK, S. M.; SHKOLNIL, J. SH.; ORLOW, W. W.: Cement Wapno Gips, 12, pp. 355-359 (1975)

(33) GLASSER, F. P.: Br. Ceram. Trans. J., 89, pp. 195-202 (1990).

(34) KONDO, R; DAIMON, M; SONG, C. T.; JINAWATH, S.: Ceram. Bull., Vol 59, n. 8 pp. $848-851$ (1980)

(35) DUTTA, D. K.; BORTHAKUR, P. C.: Cem. Concr. Res. Vol 20, pp. 711-722 (1990)

(36) VOINOVITCH,; DRON, R.: Silic. Ind., 41, pp. $209-212$ (1976)

(37) NARANG, K. C.; CHOPRA, S. K.: Silic. Ind. n. 9 9, pp 175-182 (1983)

(38) DOUGLAS, E; BRANDSTETR, J.: Cem. Concr. Res. Vol 20 pp. 746-756 (1990)

(39) REGOURD, M.: VIII Congreso Internacional de la Química del Cemento (Rio de Janeiro), Tema III,pp. 9-26 (1986)

(40) SMITH, M. A.; OSBORNE, G. J.: World Cement Technology. pp. $223-233$ (1977)

(41) DIMITRIEV, V. A.; AKUNOW, V. I.; ALBAC, V. M.; MAKASHEV, S. D.; CUKANOVA, N. V.: C.R. 10, pp. 18-19 (1981)

(42) BLUNK, G; BRAND, J; KOLLO, H; LUDWIG, U.: Zement-Kalk-Gips, 41, 12, 616 (1988)

\section{publicación del ICCET/CSIC}

\section{bases para el diseño solar pasivo}

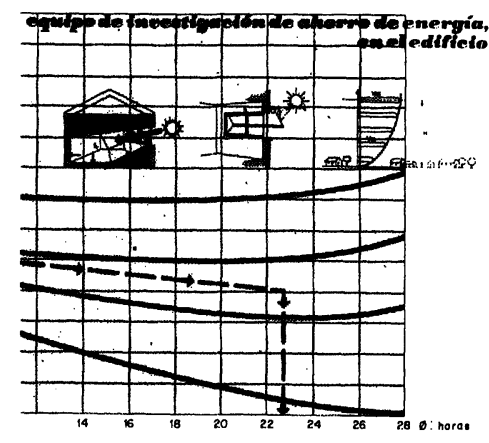

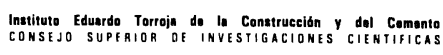

Equipo de Ahorro de Energía en el edificio

Dirección y coordinación:

Arturo García Arroyo

M. ${ }^{a}$ José Escorihuela

José Luis Esteban

José Miguel Frutos

Manuel Olaya

Bernardo Torroja
Las dificultades de suministro $y$ el alto coste de los productos energéticos convencionales han despertado la atención de los usuarios, técnicos e industriales de la edificación hacia los procedimientos y sistemas en que se basa
el aprovechamiento de otras fuentes alternael aprovechamiento de otras fuentes alterna-
tivas de energia, principalmente la solar. Esto ha generado un rápido desarrollo industrial comercial que, en opinión de los autores de este libro arrastran los siguientes defectos: este libro, arrastran un mimetico tecnologismo respecto de los sistemas convencionales que violenta las peculiaridades de la energía solar (baja densidad y variabilidad en el tiempol, y una escasa selectividad en la aplicación de los sistemas y procedimientos pasivos dando origen a un ecumenismo arquitectónico solar, al margen de las condiciones climáticas y funcionales especificas de cada caso y lugar.

En este libro, utilizando criterios y metodologia pedagógicos, se dan los fundamentos e instrumentos teórico-prácticos necesarios para el planteamiento de todo proyecto arquitectónico solar pasivo, de acuerdo con los principios éticos y económicos de conservación y ahorro de enérgia. Es decir: respeto de los presupuestos bioclimáticos, búsqueda de la máxima captación y acumulación de la radiación solar, y esmero en el aislamiento térmico de los cerramientos.

Un volumen encuadernado en cartulina ibiza plastificada, a cinco colores, de $16 \times 23 \mathrm{~cm}$, compuesto de 216 páginas, 217 figuras, 87 gráficos, 19 tablas y 10 cuadros. 